\title{
Molecular gas masses of gamma-ray burst host galaxies
}

\author{
Michał J. Michałowski ${ }^{1,2}$, A. Karska ${ }^{3}$, J. R. Rizzo ${ }^{4}$, M. Baes ${ }^{5}$, A. J. Castro-Tirado ${ }^{6}$, J. Hjorth ${ }^{7}$, L. K. Hunt ${ }^{8}$,
} P. Kamphuis ${ }^{9,10}$, M. P. Koprowski ${ }^{11}$, M. R. Krumholz ${ }^{12}$, D. Malesani ${ }^{7}$, A. Nicuesa Guelbenzu ${ }^{13}$, J. Rasmussen ${ }^{7,14}$, A. Rossi ${ }^{15}$, P. Schady ${ }^{16}$, J. Sollerman ${ }^{17}$, and P. van der Werf ${ }^{18}$

1 Astronomical Observatory Institute, Faculty of Physics, Adam Mickiewicz University, ul. Słoneczna 36, 60-286 Poznań, Poland e-mail: mj .michalowski@gmail.com

2 SUPA (Scottish Universities Physics Alliance), Institute for Astronomy, University of Edinburgh, Royal Observatory, Blakford Hill, Edinburgh, EH9 3HJ, UK

3 Centre for Astronomy, Faculty of Physics, Astronomy and Informatics, Nicolaus Copernicus University, Grudziądzka 5, 87-100 Toruń, Poland

${ }^{4}$ Centro de Astrobiología (INTA-CSIC), Ctra. M-108, km. 4, 28850 Torrejón de Ardoz, Madrid, Spain

5 Sterrenkundig Observatorium, Universiteit Gent, Krijgslaan 281-S9, 9000 Gent, Belgium

${ }^{6}$ Instituto de Astrofísica de Andalucía (IAA-CSIC), Glorieta de la Astronomía s/n, 18008 Granada, Spain

7 Dark Cosmology Centre, Niels Bohr Institute, University of Copenhagen, Juliane Maries Vej 30, 2100 Copenhagen Ø, Denmark

8 INAF-Osservatorio Astrofisico di Arcetri, Largo E. Fermi 5, 50125 Firenze, Italy

9 Astronomisches Institut der Ruhr-Universität Bochum (AIRUB), Universitätsstrasse 150, 44801 Bochum, Germany

10 National Centre for Radio Astrophysics, TIFR, Ganeshkhind, Pune 411007, India

11 Centre for Astrophysics Research, University of Hertfordshire, College Lane, Hatfield AL10 9AB, UK

12 Research School of Astronomy and Astrophysics, Australian National University, Canberra, ACT, Australia

13 Thüringer Landessternwarte Tautenburg, Sternwarte 5, 07778 Tautenburg, Germany

14 Technical University of Denmark, Department of Physics, Fysikvej, Building 309, 2800 Kgs. Lyngby, Denmark

15 INAF-OAS, via Piero Gobetti 93/3, 40129 Bologna, Italy

16 Max-Planck-Institut für Extraterrestrische Physik, Giessenbachstraße, 85748 Garching bei München, Germany

17 The Oskar Klein Centre, Department of Astronomy, AlbaNova, Stockholm University, 10691 Stockholm, Sweden

18 Leiden Observatory, Leiden University, PO Box 9513, 2300 RA Leiden, The Netherlands

Received 17 April 2018 / Accepted 3 July 2018

\section{ABSTRACT}

Context. Long gamma-ray bursts (GRBs) can potentially be used as a tool to study star formation and recent gas accretion onto galaxies. However, the information about gas properties of GRB hosts is scarce. In particular, very few carbon monoxide (CO) line detections of individual GRB hosts have been reported. It has also been suggested that GRB hosts have lower molecular gas masses than expected from their star formation rates (SFRs).

Aims. The objectives of this paper are to analyse molecular gas properties of the first substantial sample of GRB hosts and test whether they are deficient in molecular gas.

Methods. We obtained CO(2-1) observations of seven GRB hosts with the APEX and IRAM $30 \mathrm{~m}$ telescopes. We analysed these data together with all other hosts with previous $\mathrm{CO}$ observations. From these observations we calculated the molecular gas masses of these galaxies and compared them with the expected values based on their SFRs and metallicities.

Reults. We obtained detections for 3 GRB hosts $(980425,080207$, and 111005A) and upper limits for the remaining 4 (031203, 060505, 060814, and 100316D). In our entire sample of 12 CO-observed GRB hosts, 3 are clearly deficient in molecular gas, even taking into account their metallicity $(980425,060814$, and 080517). Four others are close to the best-fit line for other star-forming galaxies on the SFR- $M_{\mathrm{H}_{2}}$ plot $(051022,060505,080207$, and 100316D). One host is clearly molecule rich (111005A). Finally, the data for 4 GRB hosts are not deep enough to judge whether they are molecule deficient $(000418,030329,031203$, and 090423). The median value of the molecular gas depletion time, $M_{\mathrm{H}_{2}} / \mathrm{SFR}$, of GRB hosts is $\sim 0.3$ dex below that of other star-forming galaxies, but this result has low statistical significance. A Kolmogorov-Smirnov test performed on $M_{\mathrm{H}_{2}} /$ SFR shows an only $\sim 2 \sigma$ difference between GRB hosts and other galaxies. This difference can partly be explained by metallicity effects, since the significance decreases to $\sim 1 \sigma$ for $M_{\mathrm{H}_{2}} /$ SFR versus metallicity.

Conclusions. We found that any molecular gas deficiency of GRB hosts has low statistical significance and that it can be attributed to their lower metallicities; and thus the sample of GRB hosts has molecular properties that are consistent with those of other galaxies, and they can be treated as representative star-forming galaxies. However, the molecular gas deficiency can be strong for GRB hosts if they exhibit higher excitations and/or a lower CO-to- $\mathrm{H}_{2}$ conversion factor than we assume, which would lead to lower molecular gas masses than we derive. Given the concentration of atomic gas recently found close to GRB and supernova sites, indicating recent gas inflow, our results about the weak molecular deficiency imply that such an inflow does not enhance the SFRs significantly, or that atomic gas converts efficiently into the molecular phase, which fuels star formation. Only if the analysis of a larger GRB host sample reveals molecular deficiency (especially close to the GRB position) would this support the hypothesis of star formation that is directly fuelled by atomic gas.

Key words. ISM: lines and bands - ISM: molecules - galaxies: ISM - galaxies: star formation - gamma-ray burst: general radio lines: galaxies 


\section{Introduction}

Long gamma-ray bursts (GRBs) have long been confirmed to be the endpoints of lives of very massive stars (e.g. Hjorth et al. 2003; Stanek et al. 2003; Hjorth \& Bloom 2012). Most of the tracers of the star formation rate (SFR) of galaxies are connected with emission from massive stars (e.g. Kennicutt 1998), so that GRBs were also used to measure the star formation history of the Universe (Yüksel et al. 2008; Kistler et al. 2009; Butler et al. 2010; Elliott et al. 2012; Robertson \& Ellis 2012; Perley et al. 2016a,b). This approach is valid if GRB hosts are representative star-forming galaxies at a given redshift (Michałowski et al. 2012; Hunt et al. 2014a; Schady et al. 2014; Greiner et al. 2015; Kohn et al. 2015), or if biases are known and can be corrected for (Perley et al. 2013, 2015, 2016a,b; Boissier et al. 2013; Vergani et al. 2015; Schulze et al. 2015; Greiner et al. 2016). Gas is the fuel of star formation, so one of the important aspects of this issue is whether GRB hosts exhibit normal gas properties with respect to other star-forming galaxies.

The information about gas properties of GRB hosts is scarce. Michałowski et al. (2015) and Arabsalmani et al. (2015) provided the only measurements so far of the atomic gas properties of five such galaxies. This led to a suggestion that GRB hosts have experienced recent inflows of atomic gas. A resulting possibility of using GRBs to select galaxies for the study of gas accretion is important because the rate of the gas accretion onto galaxies is surprisingly constant since $z \sim 5$, which is at odds with the significantly changing SFR volume density of the Universe (Spring \& Michałowski 2017). Moreover, a fraction of star formation in GRB hosts may be directly fuelled by atomic gas (Michałowski et al. 2015, 2016). The existence of this process is controversial, but it has been predicted theoretically (Glover \& Clark 2012; Krumholz 2012; Hu et al. 2016; Elmegreen 2018) and is supported by some observations (Bigiel et al. 2010; Fumagalli \& Gavazzi 2008; Elmegreen et al. 2016).

Clearly, most of the star formation in the Universe is fuelled by molecular gas (Fumagalli et al. 2009; Carilli \& Walter 2013; Rafelski et al. 2016). There were several unsuccessful searches of CO lines for GRB hosts (Kohno et al. 2005; Endo et al. 2007; Hatsukade et al. 2007, 2011; Stanway et al. 2011) and only four detections so far, for the hosts of GRB 980425 (Michałowski et al. 2016), 051022 (Hatsukade et al. 2014), 080517 (Stanway et al. 2015b), and 080207 (Arabsalmani et al. 2018). These studies resulted in mixed conclusions on whether GRB hosts are deficient in molecular gas with respect to the SFR- $M_{\mathrm{H}_{2}}$ correlation of other star-forming galaxies.

Hence, the objectives of this paper are (i) to analyse molecular gas properties of the first substantial sample of GRB hosts; and (ii) to test whether these hosts are deficient in molecular gas. For this, we combined existing literature data with new observations using the APEX and IRAM $30 \mathrm{~m}$ telescopes.

We use a cosmological model with $H_{0}=70 \mathrm{~km} \mathrm{~s}^{-1} \mathrm{Mpc}^{-1}$, $\Omega_{\Lambda}=0.7$, and $\Omega_{\mathrm{m}}=0.3$. We also assume the Chabrier (2003) initial mass function (IMF), to which all star formation rates (SFRs) and stellar masses were converted (by dividing by 1.8) if given originally assuming the Salpeter (1955) IMF.

\section{Target selection and data}

\subsection{APEX}

We selected the host galaxies of all known GRBs at $z<0.12$ in the southern hemisphere (i.e. the sample with $\mathrm{H}$ I observations from Michałowski et al. 2015). These criteria were
Table 1. Log of APEX observations.

\begin{tabular}{|c|c|c|c|}
\hline GRB & Obs. date & Time/h & $\mathrm{pwv} / \mathrm{mm}$ \\
\hline \multirow{6}{*}{980425 Center } & Total & 4.04 & \\
\hline & 2015 Aug. 29 & 0.70 & $1.64-1.70$ \\
\hline & 2015 Sep. 12 & 0.30 & $0.75-0.85$ \\
\hline & 2015 Sep. 16 & 0.70 & $1.43-1.57$ \\
\hline & 2015 Oct. 31 & 1.17 & $1.22-1.96$ \\
\hline & 2015 Nov. 01 & 1.17 & $0.66-0.85$ \\
\hline \multirow[t]{4}{*}{$980425 \mathrm{WR}$} & Total & 6.57 & \\
\hline & 2015 Nov. 02 & 2.17 & $0.75-3.48$ \\
\hline & 2016 Apr. 03 & 0.10 & $2.02-2.15$ \\
\hline & 2016 Apr. 04 & 4.30 & $3.33-5.23$ \\
\hline 031203 & 2015 Sep. 10 & 0.80 & $0.83-0.91$ \\
\hline \multirow[t]{7}{*}{060505} & Total & 7.00 & \\
\hline & 2015 Aug. 28 & 1.20 & $1.50-1.67$ \\
\hline & 2015 Aug. 29 & 1.40 & $1.38-1.62$ \\
\hline & 2015 Sep. 02 & 1.40 & $1.55-1.86$ \\
\hline & 2015 Sep. 03 & 1.00 & $3.36-3.61$ \\
\hline & 2015 Sep. 04 & 1.00 & $2.50-2.73$ \\
\hline & 2015 Sep. 06 & 1.00 & $2.45-3.40$ \\
\hline \multirow[t]{4}{*}{$100316 \mathrm{D}$} & Total & 6.58 & \\
\hline & 2015 Aug. 28 & 2.11 & $1.50-1.62$ \\
\hline & 2015 Sep. 02 & 1.67 & $1.32-1.93$ \\
\hline & 2015 Sep. 06 & 2.80 & $2.45-4.80$ \\
\hline \multirow[t]{4}{*}{ 111005A Center } & Total & 1.65 & \\
\hline & 2015 Sep. 01 & 0.75 & $1.00-1.21$ \\
\hline & 2015 Sep. 12 & 0.20 & $0.72-0.84$ \\
\hline & 2015 Sep. 15 & 0.70 & $0.64-0.82$ \\
\hline \multirow[t]{5}{*}{$111005 \mathrm{~A} \mathrm{NW}$} & Total & 3.20 & \\
\hline & 2015 Sep. 17 & 0.50 & $1.52-1.61$ \\
\hline & 2016 Apr. 02 & 1.00 & $2.15-2.47$ \\
\hline & 2016 Apr. 03 & 0.60 & $1.96-2.31$ \\
\hline & 2016 Jun. 10 & 1.60 & $2.98-3.34$ \\
\hline \multirow[t]{4}{*}{$111005 \mathrm{~A} \mathrm{SE}$} & Total & 2.20 & \\
\hline & 2015 Sep. 17 & 0.50 & $1.55-1.65$ \\
\hline & 2016 Jun. 10 & 0.60 & $3.12-3.32$ \\
\hline & 2016 Jun. 11 & 1.60 & $2.49-2.83$ \\
\hline
\end{tabular}

fulfilled by GRB 980425 (the central pointing was published separately in Michałowski et al. 2016), 031203, 060505, $100316 \mathrm{D}$, and 111005A. We performed $\mathrm{CO}(2-1)$ observations using the Swedish Heterodyne Facility Instrument (SHeFI; Vassilev et al. 2008; Belitsky et al. 2006) and the SwedishESO PI Instrument for APEX (SEPIA; Belitsky et al. 2018; only for the GRB 031203 host) mounted at the Atacama Pathfinder Experiment (APEX; Güsten et al. 2006) (project no. 096.D-0280, 096.F-9302 and 097.F-9308, PI: M. Michałowski). Table 1 shows the observation log with total on-source integration times. Two and three positions were observed for the host of GRB 980425 and 111005A, respectively. The remaining galaxies are smaller than the beam $\left(\sim 27^{\prime \prime}\right)$. All observations were carried out in the on-off pattern and position-switching mode. The fluxes were corrected using the main beam efficiency of 0.75 . We reduced and analysed the data using the Continuum and Line Analysis Single Dish Software (CLAss) package within the Grenoble Image and Line Data Analysis Software ${ }^{1}$ (GILDAs; Pety 2005).

1 http://wWW.iram.fr/IRAMFR/GILDAS 
Table 2. $\log$ of IRAM $30 \mathrm{~m}$ observations.

\begin{tabular}{llcc}
\hline \hline GRB & Obs. date & Time/h & $\tau_{225 \mathrm{GHz}}$ \\
\hline 060814 & Total & 13.10 & \\
& 2017 Feb. 01 & 0.40 & 0.29 \\
& 2017 Feb. 03 & 1.60 & $0.08-0.23$ \\
& 2017 Feb. 04 & 3.20 & $0.23-0.51$ \\
& 2017 Feb. 07 & 1.40 & $0.20-0.39$ \\
& 2017 Apr. 06 & 0.70 & $0.13-0.17$ \\
& 2017 Apr. 07 & 2.00 & $0.12-0.20$ \\
& 2017 Apr. 08 & 2.20 & $0.15-0.19$ \\
& 2017 Apr. 09 & 1.60 & $0.10-1.60$ \\
\hline 080207 & Total & 17.80 & \\
& 2017 Feb. 01 & 1.60 & $0.28-0.37$ \\
& 2017 Apr. 11 & 1.90 & $0.27-0.36$ \\
& 2017 Apr. 12 & 3.70 & $0.23-0.48$ \\
& 2017 Apr. 13 & 4.30 & $0.20-0.44$ \\
& 2017 Apr. 14 & 3.30 & $0.22-0.41$ \\
& 2017 May. 22 & 3.00 & $0.24-0.36$ \\
\hline
\end{tabular}

\subsection{IRAM $30 \mathrm{~m}$}

We selected all GRB hosts in the northern hemisphere with infrared or radio detections (Hunt et al. 2014a; Perley et al. 2015; Michałowski et al. 2015) and $z>1.5$, so that the line is located at lower frequencies and easier to observe. This was fulfilled by GRB 060814 and 080207 . We performed observations with the IRAM 30 m telescope (project no. 172-16, PI: M. Michałowski) using the Eight MIxer Receiver ${ }^{2}$ (EMIR; Carter et al. 2012). We implemented wobbler-switching mode (with the offset to the reference positions of $60^{\prime \prime}$ ), which provides stable and flat baselines and optimises the total observing time. An intermediate frequency (IF) covered the frequency of the $\mathrm{CO}(2-1)$ line. We used the Fourier Transform Spectrometers 200 (FTS-200) providing $195 \mathrm{kHz}$ spectral resolution (corresponding to $\sim 0.8 \mathrm{~km} \mathrm{~s}^{-1}$ at the frequency of $\mathrm{CO}(2-1)$ of our targets) and $16 \mathrm{GHz}$ bandwidth in each linear polarisation. The observations were divided into 6 min scans, each consisting of 12 scans $30 \mathrm{~s}$ long. The pointing was verified every $1-2 \mathrm{~h}$. The observing log is presented in Table 2 with total on-source integration times. The observations were carried out during good atmospheric conditions, and the opacity $\left(\tau_{225 \mathrm{GHz}}\right)$ was uniform across different runs. We reduced the data using the Class package within GiLdas (Pety 2005). Each spectrum was calibrated, and corrected for baseline shape. The spectra were aligned in frequency and noise-weight averaged. Some well-known platforming, due to the fact that the instantaneous bandwidth of $4 \mathrm{GHz}$ is sampled by three different FTS units, was corrected off-line by a dedicated procedure within Class. In all cases, the CO line is far away from the step of the platforming.

\subsection{Literature data for additional GRB hosts}

In addition to the $\mathrm{CO}(2-1)$ measurements obtained here, we included all other $\mathrm{CO}$ measurements for GRB hosts from the literature. All molecular masses were converted into $\alpha_{\mathrm{CO}}=5 M_{\odot}\left(\mathrm{K} \mathrm{km} \mathrm{s}^{-1} \mathrm{pc}^{2}\right)^{-1}$ and to the line luminosity ratios in temperature units $L_{2-1}^{\prime} / L_{1-0}^{\prime}=0.5, L_{3-2}^{\prime} / L_{1-0}^{\prime}=0.27$, or $L_{4-3}^{\prime} / L_{1-0}^{\prime}=0.17$ (the Milky Way values, see Table 2 of

\footnotetext{
2 www.iram.es/IRAMES/mainWiki/EmirforAstronomers
}

Carilli \& Walter 2013) if these masses were based on $\mathrm{CO}(2-1)$, $\mathrm{CO}(3-2)$, or $\mathrm{CO}(4-3)$ observations, respectively. These assumed line ratios are conservatively low, so that they lead to conservatively high $M_{\mathrm{H}_{2}}$. We are therefore able to robustly test for any molecular deficiency of GRB hosts.

We included the hosts of GRB 000418 (Hatsukade et al. 2011), for which we converted the $M_{\mathrm{H}_{2}}$ upper limit from $L_{2-1}^{\prime} / L_{1-0}^{\prime}=1$ into 0.5 and from $\alpha_{\mathrm{CO}}=0.8 M_{\odot}\left(\mathrm{K} \mathrm{km} \mathrm{s}^{-1} \mathrm{pc}^{2}\right)^{-1}$ to 5; of GRB 030329 (Kohno et al. 2005; Endo et al. 2007), for which we converted the $M_{\mathrm{H}_{2}}$ upper limit from $\alpha_{\mathrm{CO}}=40 M_{\odot}\left(\mathrm{K} \mathrm{km} \mathrm{s}^{-1} \mathrm{pc}^{2}\right)^{-1}$ into 5; of GRB 051022 (Hatsukade et al. 2014), for which we converted the $M_{\mathrm{H}_{2}}$ detection from $L_{4-3}^{\prime} / L_{1-0}^{\prime}=0.85$ into 0.17 and from $\alpha_{\mathrm{CO}}=4.3 M_{\odot}\left(\mathrm{K} \mathrm{km} \mathrm{s}^{-1} \mathrm{pc}^{2}\right)^{-1}$ into 5; of GRB 080517 (Stanway et al. 2015b), for which we converted the $M_{\mathrm{H}_{2}}$ detection from $\alpha_{\mathrm{CO}}=4.3 M_{\odot}\left(\mathrm{K} \mathrm{km} \mathrm{s}^{-1} \mathrm{pc}^{2}\right)^{-1}$ into 5; and of GRB 090423 (Stanway et al. 2011), for which we converted the $M_{\mathrm{H}_{2}}$ detection from $L_{3-2}^{\prime} / L_{1-0}^{\prime}=1$ into 0.27 and from $\alpha_{\mathrm{CO}}=0.8 M_{\odot}$ $\left(\mathrm{K} \mathrm{km} \mathrm{s}^{-1} \mathrm{pc}^{2}\right)^{-1}$ into 5 .

We did not use the CO(3-2) observations of GRB 980425 of Hatsukade et al. (2007) because our deeper data resulted in a detection. Moreover, we excluded GRB 020819B because the low-redshift galaxy with the existing $\mathrm{CO}$ measurement (Hatsukade et al. 2014) has been shown not to be related to the GRB (Perley et al. 2017b). For the GRB 080207 host, the $\mathrm{CO}(3-2)$ line observations were recently reported by Arabsalmani et al. (2018). We did not use these values in subsequent analysis, because our lower transition likely traces a larger fraction of the total molecular gas content. We note, however, that the obtained gas masses are consistent (see Sect. 3).

For all GRB hosts in our CO sample we used the literature values for their redshifts, SFRs and metallicities, as listed in Table A.1. For the host of GRB 060814, we calculated the metallicity based on the $R_{23}$ method of Kobulnicky \& Kewley (2004) based on the [O II], [O III], and $\mathrm{H} \beta$ emission lines, using the fluxes reported in Krühler et al. (2015). We obtained $12+\log (\mathrm{O} / \mathrm{H}) \sim 8.38 \pm 0.35$.

Additionally, we included values measured for the host of SN 2009bb, the relativistic supernova (SN) type Ic (Michałowski et al. 2018b) and plot them in Figs. 2 and 4. SNe of this type may have similar engines as GRBs, but no $\gamma$-rays were detected. Therefore we did not use it for the statistical analysis quoted for GRB hosts, and it does not appear in Figs. 3 and 5.

\subsection{Other galaxy samples}

In order to place the GRB hosts in the context of general galaxy populations, we compared their properties with those of the following galaxy samples, chosen based on the availability of the gas mass estimates: the optical-flux-limited spirals and irregulars with IRAS data (Young et al. 1989), local luminous infrared galaxies (LIRGs; Sanders et al. 1991), local ultra-luminous infrared galaxies (ULIRGs; Solomon et al. 1997), the Herschel Reference Survey (HRS; Boselli et al. 2010, 2014; Cortese et al. 2012, 2014; Ciesla et al. 2014), $\mathrm{H}$ I-dominated, low-mass galaxies and large spiral galaxies (Leroy et al. 2008), $0.01<z<0.03$ mass-selected galaxies with $8.5<\log \left(M_{\star} / M_{\odot}\right)<10$ (Bothwell et al. 2014), $0.025<z<0.2$ mass-selected galaxies with $\log \left(M_{\star} / M_{\odot}\right)>10$ and infrared detections (Bertemes et al. 2018), metal-poor dwarfs (Hunt et al. 2014b, 2015, 2017; Leroy et al. 2007), metal-poor dwarfs from the Herschel Dwarf Galaxy Survey (Madden et al. 2013; Cormier et al. 2014), Virgo-cluster dwarfs (Grossi et al. 2016), $z \sim 1.5$ BzK galaxies (Daddi et al. 2010; Magdis et al. 
2011; Magnelli et al. 2012), and $1.2<z<4.1$ submm galaxies (Bothwell et al. 2013; Michałowski et al. 2010).

All SFRs were converted into the Chabrier (2003) IMF. The molecular masses were converted into $\alpha_{\mathrm{CO}}=5 M_{\odot}\left(\mathrm{K} \mathrm{km} \mathrm{s}^{-1} \mathrm{pc}^{2}\right)^{-1}$ and to the Milky Way line ratios if they were based on higher $\mathrm{CO}$ transitions. Namely, Bothwell et al. (2014), Daddi et al. (2010) and Leroy et al. (2008) assumed $L_{2-1}^{\prime} / L_{1-0}^{\prime}=1,0.16$, and 0.8 respectively, and Hunt et al. (2014b) assumed $L_{3-2}^{\prime} / L_{1-0}^{\prime}=0.6$. The Galactic value of $\alpha_{C O}$ is appropriate for $0.4-1$ solar metallicity galaxies discussed here (Bolatto et al. 2013; Hunt et al. 2014b). Following Hunt et al. (2015), metallicities from Bothwell et al. (2014) were converted from the calibration of Kewley \& Dopita (2002, KD02) into that of Pettini \& Pagel (2004, PP04 N2) using the equation derived by Kewley \& Ellison (2008, their Table 3).

Even though SFR estimates of other galaxies are often derived from various diagnostics (ultraviolet, $\mathrm{H} \alpha$, infrared, and radio), they are broadly consistent (Salim et al. 2007; Wijesinghe et al. 2011; Davies et al. 2016; Wang et al. 2016), even in dwarf galaxies, except at very low $\mathrm{SFR}<0.001 M_{\odot} \mathrm{yr}^{-1}$ (Huang et al. 2012; Lee et al. 2009), not discussed here.

\section{Results}

The positions of our APEX and IRAM $30 \mathrm{~m}$ pointings and the obtained $\mathrm{CO}(2-1)$ spectra are shown in Fig. 1. The spectra were binned to a velocity resolution of $20 \mathrm{~km} \mathrm{~s}^{-1}$, except for the GRB 080207 host, for which $50 \mathrm{~km} \mathrm{~s}^{-1}$ channels were adopted. The derived parameters are shown in Table 3 . The fluxes were integrated within the velocity ranges shown in Fig. 1 as vertical dotted lines. They were chosen to encompass the full extent of the lines for the detected targets, and the most significant positive feature within the velocity range from -300 to $300 \mathrm{~km} \mathrm{~s}^{-1}$ relative to the optical redshift for the non-detected targets in order to obtain the most conservative upper limits. For these nondetected targets we integrated the spectra in the region of a width of $200 \mathrm{~km} \mathrm{~s}^{-1}$, likely to be the velocity width of such galaxies, and of $50 \mathrm{~km} \mathrm{~s}^{-1}$ for the WR region, as it is unlikely that this pointing traces gas at a wider range of velocities (see Fig. 3 of Christensen et al. 2008). The $\mathrm{CO}(2-1)$ line luminosities were calculated using Eq. (3) in Solomon et al. (1997) and converted into the $\mathrm{CO}(1-0)$ luminosities assuming $L_{1-0}^{\prime}=2 \times L_{2-1}^{\prime}$. The Galactic CO-to- $\mathrm{H}_{2}$ conversion factor $\alpha_{\mathrm{CO}}=5 M_{\odot} / \mathrm{K} \mathrm{km} \mathrm{s}^{-1} \mathrm{pc}^{2}$ was used to calculate molecular gas masses $\left(M_{\mathrm{H}_{2}}=\alpha_{\mathrm{CO}} L_{1-0}^{\prime}\right)$.

\subsection{SFR vs. $M_{\mathrm{H}_{2}}$}

The infrared luminosity (or SFR) as a function of CO line luminosity (or $M_{\mathrm{H}_{2}}$ ) for GRB hosts and other galaxies is shown in Fig. 2. The best linear fit in log-log space to all non-GRB galaxies with SFRs lower than those of ULIRGs $\left(\mathrm{SFR}<100 M_{\odot} \mathrm{yr}^{-1}\right)$ is (the solid line in Fig. 2)

$\log \left(\mathrm{SFR} / M_{\odot} \mathrm{yr}^{-1}\right)=0.95 \times \log \left(M_{\mathrm{H}_{2}} / M_{\odot}\right)-8.57$.

The scatter around this relation is $\sim 0.42$ dex. When ULIRGs are included, this equation changes to (the dashed line in Fig. 2)

$\log \left(\mathrm{SFR} / M_{\odot} \mathrm{yr}^{-1}\right)=1.10 \times \log \left(M_{\mathrm{H}_{2}} / M_{\odot}\right)-9.96$.

As reported in Michałowski et al. (2016), we found a low molecular gas content in the GRB 980425 host given its SFR. Similarly, the hosts of GRB 100316D and 060814 are deficient in $M_{\mathrm{H}_{2}}$ given their SFRs. Our $M_{\mathrm{H}_{2}}$ upper limit for the GRB 031203 host is $\sim 0.5$ dex higher than the value suggested by the bestfit relation of Eq. (1) so that we cannot conclude much about its molecular gas content. Wiersema et al. (2018) measured a molecular gas mass $\sim 5$ times lower than our upper limit based on the $\mathrm{H}_{2}$ 0-0 S(7) rotational emission line. Our $M_{\mathrm{H}_{2}}$ upper limit for the GRB 060505 host is not sufficiently strong to test for any molecular gas deficiency, but it is close to the best-fit line for other star-forming galaxies, which means that this galaxy is not richer in molecular gas than the average of other galaxies. We found that the GRB 080207 host is very close to the best-fit line for other galaxies on the SFR- $M_{\mathrm{H}_{2}}$ diagram, consistent with the results of Arabsalmani et al. (2018) based on the $\mathrm{CO}(3-2)$ line. The host of GRB $111005 \mathrm{~A}$ is molecule rich with $\log \left(M_{\mathrm{H}_{2}} / \mathrm{SFR} \mathrm{yr}^{-1}\right) \sim 9.34$, that is, $\sim 0.24$ dex above the best-fit relation for other galaxies at the relevant SFR. Consistently with Michałowski et al. (2018b), we show that the host of SN 2009bb has a molecular gas mass that is a few times lower than its SFR suggests.

The second pointing for the GRB 980425 host, towards the Wolf-Rayet (WR) region (for its properties, see Hammer et al. 2006; Le Floc'h et al. 2006, 2012; Christensen et al. 2008; Michałowski et al. 2009, 2014, 2016; Krühler et al. 2017) resulted in an upper limit close to the best-fit line. While we cannot establish any molecular deficiency for this region, it is therefore definitely not molecule rich, in contrast with its high abundance of atomic gas (Arabsalmani et al. 2015). Both the central and NW regions of the GRB 111005A host are molecule rich, but the SE region is at least slightly molecule deficient, given its $\mathrm{CO}$ upper limit.

Because of our choice to adopt the Milky Way CO line ratios instead of those of M 82 (see Sect. 2.3), we obtained a molecular gas mass that is approximately five times higher for the GRB 051022 host, and hence its molecular gas deficiency is not as dramatic as presented originally in Hatsukade et al. (2014), but still apparent (Fig. 2). Our correction for the GRB 080517 is small with respect to the values used in Stanway et al. (2015b), so we recover its reported molecular gas deficiency.

The revised, lower value of the infrared luminosity of the host of GRB 000418 (compare Michałowski et al. 2008 and Perley et al. 2017b) means that the CO observations (Hatsukade et al. 2011) do not provide useful constraints on its location on the SFR- $M_{\mathrm{H}_{2}}$ diagram (see Fig. 2). Similarly, the upper limits on $L_{\mathrm{IR}}$ available for GRB 030329 (Endo et al. 2007) and 090423 (Stanway et al. 2011) do not constrain the positions of these galaxies relative to the best-fit SFR- $M_{\mathrm{H}_{2}}$ relation. Hence we did not use these three hosts with upper limits for both SFRs and $M_{\mathrm{H}_{2}}$ in the statistical analysis.

The median value of the molecular gas depletion time for non-GRB galaxies is $\log \left(M_{\mathrm{H}_{2}} / \mathrm{SFR} \mathrm{yr}^{-1}\right)=9.099_{-0.020}^{+0.031}$, whereas for GRB hosts it is $8.83_{-0.52}^{+0.24}$ (the errors were obtained by randomly perturbing 500 times the measured values within their errors and assessing the $68 \%$ confidence interval of the obtained medians), where we treated the upper limits as actual values. The value for GRB hosts therefore is an upper limit. Hence, GRB hosts have molecular gas masses $\sim 0.3$ dex below the expectations from their SFR, but this result has low significance.

The cumulative distributions of the $M_{\mathrm{H}_{2}} / \mathrm{SFR}$ ratio (molecular gas depletion time) is shown in Fig. 3. For these statistics we excluded hosts with weak upper limits (031203) and those with upper limits for both $M_{\mathrm{H}_{2}}$ and SFRs (000418, 030329, and 090423). Using the Kolmogorov-Smirnov (K-S) test, we found that we can rule out the null hypothesis that the $M_{\mathrm{H}_{2}} / \mathrm{SFR}$ values of the GRB hosts were drawn from the same distribution as those 

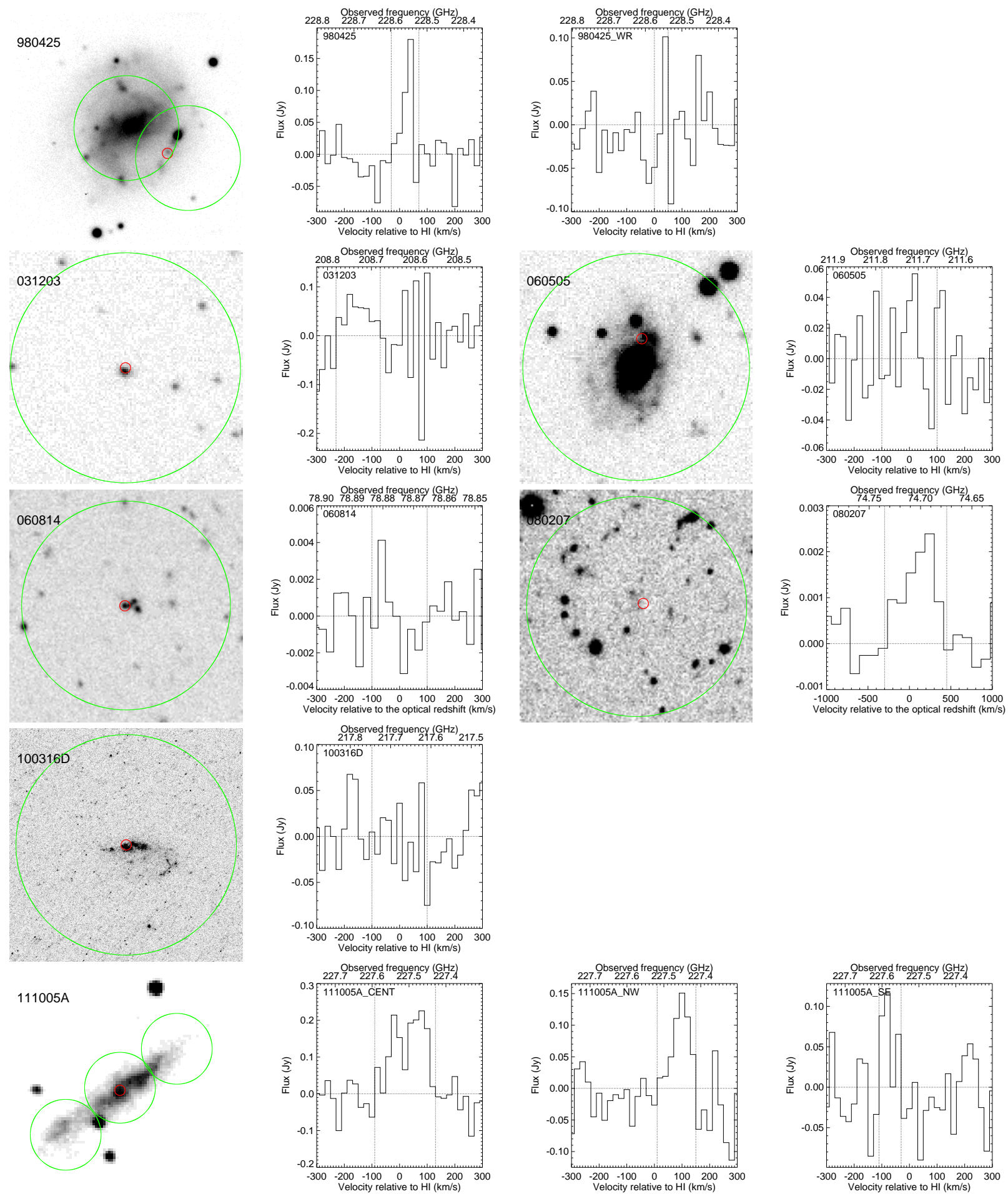

Fig. 1. For each GRB host (labelled in the top left corner of each panel), first panel: optical image (Sollerman et al. 2005; Mazzali et al. 2006; Thöne et al. 2008; Hjorth et al. 2012; Starling et al. 2011; Michałowski et al. 2018a) together with the green circles marking the positions of the pointings and the beam sizes of our $\mathrm{CO}(2-1)$ observations. GRB positions are marked with red circles. North is up and east is to the left. Other panels: corresponding $\mathrm{CO}(2-1)$ spectra. Vertical dotted lines show the velocity intervals within which the line fluxes were measured.

of other star-forming galaxies at a significance level $p=0.05$, corresponding to a difference with a low statistical significance of $\sim 1.9 \sigma$. In order to assess the influence of the measurement errors on this result, we repeated the $\mathrm{K}-\mathrm{S}$ test using the GRB values perturbed by their errors and found that the significance remains similar.

\section{2. $M_{\mathrm{H}_{2}} / S F R$ vs. metallicity}

The CO-to- $\mathrm{H}_{2}$ conversion factor is metallicity dependent (e.g. Bolatto et al. 2013), therefore we explored the $M_{\mathrm{H}_{2}} / \mathrm{SFR}$ ratio as a function of metallicity (Fig. 4). Using the galaxies with metallicity measurement, the linear fit to all non-GRB galaxies is (the 
Table 3. APEX and IRAM $30 \mathrm{~m} \mathrm{CO}(2-1)$ line fluxes and luminosities.

\begin{tabular}{|c|c|c|c|c|c|c|}
\hline $\begin{array}{l}\text { GRB } \\
\text { (1) }\end{array}$ & $\begin{array}{c}F_{\text {int }} \\
\left(\mathrm{Jy} \mathrm{km} \mathrm{s}^{-1}\right) \\
(2)\end{array}$ & $\begin{array}{r}S / N \\
(3) \\
\end{array}$ & $\begin{array}{c}F_{\mathrm{int}} \\
\left(10^{-20} \mathrm{~W} \mathrm{~m}^{-2}\right) \\
(4)\end{array}$ & $\begin{array}{c}\log L \\
\left(L_{\odot}\right) \\
(5)\end{array}$ & $\begin{array}{c}\log L^{\prime} \\
\left(\mathrm{K} \mathrm{km} \mathrm{s}^{-1} \mathrm{pc}^{2}\right) \\
(6)\end{array}$ & $\begin{array}{c}\log M_{\mathrm{H}_{2}, \mathrm{CO}} \\
\left(M_{\odot}\right) \\
(7)\end{array}$ \\
\hline 980425 & $5.65 \pm 1.27$ & 4.4 & $4.34 \pm 0.98$ & $3.27_{-0.11}^{+0.09}$ & $6.67_{-0.11}^{+0.09}$ & $7.67_{-0.11}^{+0.09}$ \\
\hline 980425_WR & $1.33 \pm 1.28$ & 1.0 & $1.02 \pm 0.98$ & $2.64_{-1.43}^{+0.29}$ & $6.04_{-1.43}^{+0.29}$ & $7.04_{-1.43}^{+0.29}$ \\
\hline 031203 & $7.51 \pm 3.35$ & 2.2 & $5.77 \pm 2.58$ & $\begin{array}{l}5.58_{-0.26}^{+0.16} \\
\text { +0.16 }\end{array}$ & $8.99_{-0.26}^{+0.16}$ & $9.99_{-0.26}^{+0.16}$ \\
\hline 060505 & $1.18 \pm 1.64$ & 0.7 & $0.91 \pm 1.26$ & $<5.21$ & $<8.62$ & $<9.62$ \\
\hline 060814 & $-0.04 \pm 0.11$ & -0.4 & $-0.03 \pm 0.09$ & $<6.51$ & $<9.92$ & $<10.92$ \\
\hline 080207 & $0.38 \pm 0.11$ & 3.5 & $0.29 \pm 0.08$ & $6.90_{-0.14}^{+0.11}$ & $10.30_{-0.14}^{+0.11}$ & $11.30_{-0.14}^{+0.11}$ \\
\hline $100316 \mathrm{D}$ & $-0.88 \pm 2.25$ & -0.4 & $-0.68 \pm 1.73$ & $<4.76$ & $<8.16$ & $<9.16$ \\
\hline 111005A_CENT & $28.49 \pm 2.94$ & 9.7 & $21.91 \pm 2.26$ & $4.35_{-0.05}^{+0.04}$ & $7.75_{-0.05}^{+0.04}$ & $8.75_{-0.05}^{+0.04}$ \\
\hline 111005A_NW & $10.27 \pm 2.13$ & 4.8 & $7.90 \pm 1.63$ & $3.90_{-0.10}^{+0.08}$ & $7.31_{-0.10}^{+0.08}$ & $8.31_{-0.10}^{+0.08}$ \\
\hline 111005A_SE & $5.41 \pm 1.52$ & 3.5 & $4.16 \pm 1.17$ & $3.62_{-0.14}^{+0.11}$ & $7.03_{-0.14}^{+0.11}$ & $8.03_{-0.14}^{+0.11}$ \\
\hline
\end{tabular}

Notes. (1) GRB. (2) Integrated flux within the velocity interval shown by the dotted lines in Fig. 1. (3) Signal-to-noise ratio of the line within this velocity interval. (4) Corresponding integrated flux in $\mathrm{W} \mathrm{m}^{-2}$. (5) Line luminosity. (6) Line luminosity in temperature units based on Eq. (3) in Solomon et al. (1997). (7) Molecular gas mass estimated assuming $L_{\mathrm{CO}(1-0)}^{\prime}=2 \times L_{\mathrm{CO}(2-1)}^{\prime}$ (see Sects. 2.3 and 3) and the Galactic CO-to- $\mathrm{H}_{2}$ conversion factor $\alpha_{\mathrm{CO}}=5 M_{\odot} \mathrm{K} \mathrm{km} \mathrm{s}^{-1} \mathrm{pc}^{2}$.

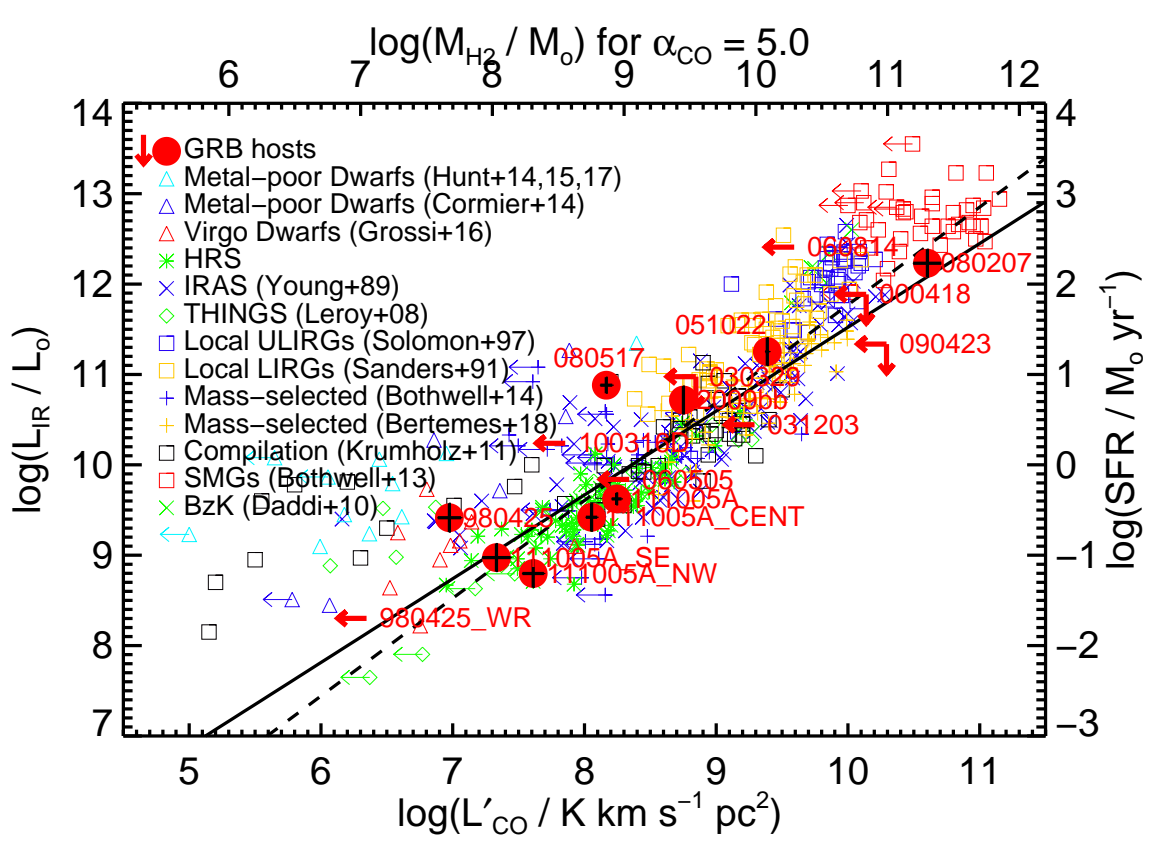

Fig. 2. Infrared luminosity or the corresponding SFR as a function of CO luminosity, or the corresponding molecular gas mass with the CO-to- $\mathrm{H}_{2}$ conversion factor $\alpha_{\mathrm{CO}}=5 M_{\odot}\left(\mathrm{K} \mathrm{km} \mathrm{s}^{-1} \mathrm{pc}^{2}\right)^{-1}$. GRB hosts are marked with full red circles or red arrows with crosses showing the errors. The symbols of other galaxies are indicated in the legend and described in Sect. 2.4. The solid black line is a linear fit to the non-GRB galaxies excluding ULIRGs Eq. (1), whereas the dashed black line represents the fit including ULIRGs Eq. (2). The $\sim 0.3$ dex shift for GRB hosts towards lower $M_{\mathrm{H}_{2}}$ is not statistically significant (see Sect. 3.1). solid line in Fig. 4)

$\log \left(M_{\mathrm{H}_{2}} / \mathrm{SFR} \mathrm{yr}^{-1}\right)=2.33 \times[12+\log (\mathrm{O} / \mathrm{H})]-11.1$.

The scatter around this relation is $\sim 0.35$ dex.

The molecular deficiency of the GRB 980425 is confirmed, even taking into account its sub-solar metallicity, that is to say, it has a shorter molecular gas depletion time than expected for its SFR and metallicity. This is at odds with the discussion in Arabsalmani et al. (2018) that this galaxy has normal molecular gas properties. However, they compared $M_{\mathrm{H}_{2}}$ with stellar mass, not SFR, as we do here, and also used the dwarf sample of Grossi et al. (2016) as a comparison, but these galaxies exhibit much lower metallicities than the GRB 980425 host (see Fig. 4). Similarly, the molecular gas deficiency of the hosts of GRB 080517 and 060814 is confirmed after taking into account their metallicities.
The hosts of GRB 051022, 080207, and 100316D have depletion times consistent with the expected values given their metallicities (the GRB 100316D host represents an upper limit, therefore we do not know whether it is close to the best-fit relation). Only the GRB 111005A host is clearly molecule rich for its metallicity. The limits for the hosts of GRB 031203 and 060505 are not constraining because they are significantly above the best fit line.

Our upper limit for the WR region of the GRB 980425 host is $\sim 0.4$ dex above the best-fit line in Fig. 4 , but the beam size of our observations is much larger than this region (Fig. 1), which means that in reality our observations also probe the highermetallicity regions.

Similarly to the results presented in Sect. 3.1, the central and NW regions of the GRB 111005A host are rich in molecular gas given their SFR and metallicity. On the other hand, the 


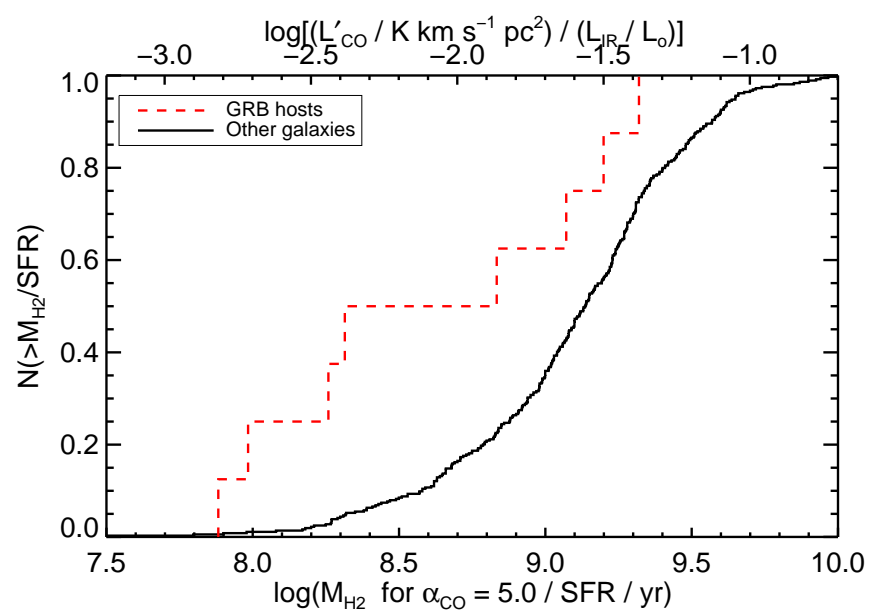

Fig. 3. Cumulative distribution of molecular gas depletion time (or the inverse of the star formation efficiency), i.e. the ratio of the CO luminosity to the infrared luminosity or the corresponding molecular gas mass with the CO-to- $\mathrm{H}_{2}$ conversion factor $\alpha_{\mathrm{CO}}=5 M_{\odot}\left(\mathrm{K} \mathrm{km} \mathrm{s}^{-1} \mathrm{pc}^{2}\right)^{-1}$ to the star formation rate (SFR). The distribution of GRB hosts is shown as the dashed red line, whereas that of other galaxies is shown as the solid black line. We treated the upper limits as actual values, so the histogram for GRB hosts is an upper limit. GRB hosts are systematically shifted to the left on this diagram (lower $M_{\mathrm{H}_{2}}$ given their SFRs), but this is not statistically significant (see Sect. 3.1).

$\mathrm{SE}$ region has a much lower molecular gas content, close to the best-fit line.

For GRB hosts, the median value of the residual from this best fit is $-0.21 \pm 0.07 \mathrm{yr}^{-1}$, where we treated the upper limits as actual values. This value is therefore an upper limit.

The cumulative distributions of residuals around the best-fit line Eq. (3) is shown in Fig. 5. For these statistics we excluded hosts with weak upper limits (031203 and 060505) and those with upper limits for both $M_{\mathrm{H}_{2}}$ and SFRs $(000418,030329$, and 090423). Using the $\mathrm{K}-\mathrm{S}$ test, we we found that we can reject the null hypothesis that the residuals around the best-fit line for GRB hosts were drawn from the same distribution as those for other star-forming galaxies only at a significance level $p=0.33$, corresponding to $a \sim 1 \sigma$ difference.

\subsection{Molecular gas fraction}

Using the $\mathrm{H}$ I data from Michałowski et al. (2015), we can constrain the molecular gas fraction $\left(M_{\mathrm{H}_{2}} /\left(M_{\mathrm{H}_{2}}+M_{\mathrm{HI}}\right)\right)$ to be $\sim 7 \%$ for the GRB 980425 host, $<15 \%$ for the GRB 060505 host, and $\sim 13 \%$ for the GRB $111005 \mathrm{~A}$ host. This is within the scatter of but on the lower side compared to other star-forming galaxies (a few to a few tens of percent ; Young et al. 1989; Devereux \& Young 1990; Leroy et al. 2008; Saintonge et al. 2011; Cortese et al. 2014; Boselli et al. 2014) and SN hosts (Galbany et al. 2017; Michałowski et al. 2018b).

\section{Discussion}

We obtained mixed results from analysing CO data for 12 GRB hosts from our survey and from the literature. Three GRB hosts are clearly deficient in molecular gas, even taking into account their metallicity $(980425,060814$, and 080517). Four others are close to the best fit-line for other star-forming galaxies in the SFR- $M_{\mathrm{H}_{2}}$ plot $(051022,060505,080207$, and 100316D). One host is clearly molecule-rich (111005A). Finally, for 4 GRB hosts the data are not deep enough to judge whether they are molecule deficient (000418, 030329, 031203, and 090423).

These results suggest that GRB hosts may be preferentially found in galaxies with lower molecular gas content than other star-forming galaxies, as there are more examples of GRB hosts in the $M_{\mathrm{H}_{2}}$-poor part of the $M_{\mathrm{H}_{2}}$-SFR diagram, and the median molecular depletion timescale $\left(M_{\mathrm{H}_{2}} / \mathrm{SFR}\right)$ of GRB hosts is $\sim 0.3$ dex shorter that of other galaxies. However, the difference between GRB hosts and other star-forming galaxies is significant only at the $\sim 2 \sigma$ level when analysing $M_{\mathrm{H}_{2}} /$ SFR (Figs. 2 and 3 ). Moreover, the statistical significance of this tentative difference decreases further to the $\sim 1 \sigma$ level when taking the metallicity into account (Figs. 4 and 5). Hence, our sample is statistically consistent with other star-forming galaxies.

Recent high-resolution observations of GRB and SN hosts showed concentrations of atomic gas close to the GRB and SN positions (Michałowski et al. 2015, 2018b; Arabsalmani et al. 2015), strongly supporting the hypothesis of recent inflow of gas at these sites. The sample of GRB/SN hosts can then be used to study recent gas inflow. Our result of a very weak molecular deficiency (if any) implies that either the SFRs of GRB/SN hosts are not significantly enhanced by such inflow, or that atomic gas is efficiently converted into the molecular phase, so that SFR and $M_{\mathrm{H}_{2}}$ increase hand in hand.

However, if molecular deficiency is confirmed with a larger sample of GRB hosts, then this will be consistent with a scenario in which their SFRs are enhanced by a recent inflow of atomic gas that did not have time to convert into the molecular phase. Moreover, a low molecular gas content would be consistent with star formation fuelled directly by atomic gas (Michałowski et al. 2015).

Two other issues need to be pointed out. First, most of our $M_{\mathrm{H}_{2}}$ estimates are based on the $\mathrm{CO}(2-1)$ line or higher transitions. In order to calculate molecular gas masses, we converted these line luminosities into those of the $\mathrm{CO}(1-0)$ line assuming a conservatively low Milky Way $L_{2-1}^{\prime} / L_{1-0}^{\prime}$ ratio, giving conservatively high $M_{\mathrm{H}_{2}}$. If however the gas in GRB hosts is even less excited than the Milky Way, then the real 2-1/1-0 ratio ratio is even lower, and our assumption would result in too low $M_{\mathrm{H}_{2}}$. This is unlikely, however, because GRB hosts are usually found to have a high SFR given their stellar masses (Castro Cerón et al. 2006, 2010; Savaglio et al. 2009; Thöne et al. 2009), which likely leads to high excitations (see Michałowski et al. 2016) and high $L_{2-1}^{\prime} / L_{1-0}^{\prime}$ ratios in turn. If this is the case generally, then our $M_{\mathrm{H}_{2}}$ are overestimated, and the difference between GRB hosts and other galaxies is stronger than suggested by our analysis. In particular, if we were to adopt the SMG or M82 2-1/1-0 ratios, then the molecular gas masses of GRB hosts would be 1.7-2.0 times lower (Table 2 of Carilli \& Walter 2013), and the difference between the GRB hosts and other galaxies would be statistically significant. This can be tested with sensitive observations of other CO transitions (especially 1-0).

Second, our tentative molecular deficiency could result from the assumption of too low $\alpha_{\mathrm{CO}}$. We did take into account the variation of $\alpha_{\mathrm{CO}}$ with metallicity (Fig. 4), but it is possible that other properties (e.g. gas density or turbulence) lead to high $\alpha_{\mathrm{CO}}$ and result in weak $\mathrm{CO}$ emission. On the other hand, if the correct $\alpha_{\mathrm{CO}}$ for GRB hosts is closer to the low value measured for starbursts (Bolatto et al. 2013), then the real molecular masses of GRB hosts are approximately five times lower than we measure and the molecular deficiency is statistically significant. This aspect is much more difficult to investigate (also for non-GRB galaxies), because there is no robust way of measuring $\alpha_{\mathrm{CO}}$, especially in non-standard environments. 


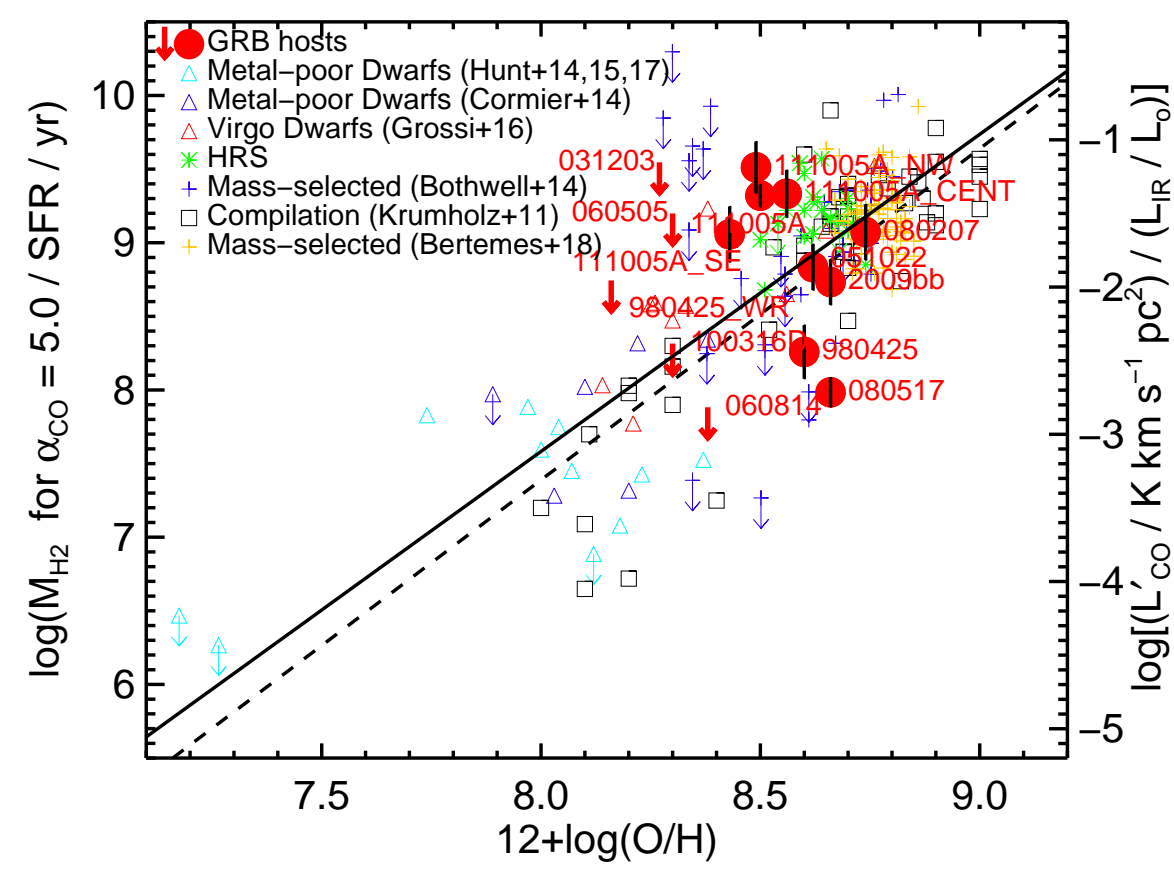

Fig. 4. Molecular gas depletion time (or the inverse of the star formation efficiency), i.e. the ratio of the $\mathrm{CO}$ luminosity to the infrared luminosity or the corresponding molecular gas mass with the CO-to- $\mathrm{H}_{2}$ conversion factor $\alpha_{\mathrm{CO}}=5 M_{\odot}\left(\mathrm{K} \mathrm{km} \mathrm{s}^{-1} \mathrm{pc}^{2}\right)^{-1}$ to the SFR as a function of metallicity. GRB hosts are marked with full red circles or red arrows with vertical bars showing the errors. The symbols of other galaxies are indicated in the legend and described in Sect. 2.4. The solid black line is our fit to the non-GRB galaxies (Eq. (3)), whereas the dashed black line is the relation found by Hunt et al. (2015). GRB hosts are consistent with other galaxies (see Sect. 3.2).

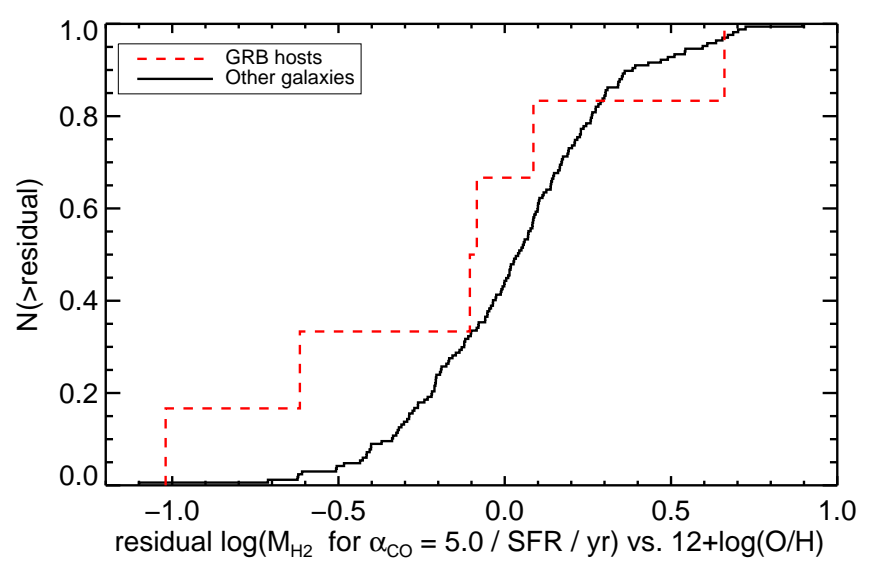

Fig. 5. Cumulative distribution of the residuals with respect to the solid line in Fig. 4 (Eq. (3)), showing the relation between metallicity and molecular gas depletion time (or the inverse of the star formation efficiency), i.e. the ratio of the $\mathrm{CO}$ luminosity to the infrared luminosity or the corresponding molecular gas mass with the $\mathrm{CO}-$ to- $\mathrm{H}_{2}$ conversion factor $\alpha_{\mathrm{CO}}=5 M_{\odot}\left(\mathrm{K} \mathrm{km} \mathrm{s}^{-1} \mathrm{pc}^{2}\right)^{-1}$ to the SFR. The distribution of GRB hosts is shown as the dashed red line, whereas that of other galaxies is shown as the solid black line. We treated the upper limits as actual values, so the histogram for GRB hosts is an upper limit. GRB hosts are systematically shifted to the left on this diagram (lower $M_{\mathrm{H}_{2}}$ given their SFRs and metallicity), but this is not statistically significant (see Sect. 3.2).

We also stress that it is important to investigate the molecular gas properties with high-resolution observations. If a molecular deficiency is found locally close to the GRB positions, then this will be consistent with star formation fuelled directly by atomic gas. In such a scenario, we are not able to capture this effect using the existing $\mathrm{CO}$ data with low spatial resolution, as the hosts on average are not significantly molecule poor.

This analysis can be improved by investigating a larger sample of GRB hosts, and possibly with deeper observations that allowing probing well below the average molecular gas depletion time of other star-forming galaxies. Moreover, the caveat of our sample is that it is heterogenous, including low- $z$ hosts and highly star-forming hosts at higher redshifts (Hunt et al. 2011, 2014a; Svensson et al. 2012; Perley et al. 2015). This demonstrates the need of obtaining CO data for a larger sample of homogeneously selected GRB hosts. This is likely possible only with ALMA, because we have targeted nearby and bright hosts with $\mathrm{CO}$ emission that is potentially easier to detect. ALMA will be able to detect fainter targets and thus will enable studies of a larger and unbiased sample.

\section{Conclusions}

We observed the $\mathrm{CO}(2-1)$ line for 7 GRB hosts, obtaining detections for 3 GRB hosts (980425, 080207, and 111005A) and upper limits for the remaining 4 (031203, 060505, 060814, and 100316D). In our entire sample of 12 CO-observed GRB hosts, including objects from the literature, 3 are clearly deficient in molecular gas, even taking into account their metallicity $(980425,060814$, and 080517). Four others are close to the best-fit line for other star-forming galaxies in the SFR- $M_{\mathrm{H}_{2}}$ plot (051022, 060505, 080207, and 100316D). One host is clearly molecule rich (111005A). Finally, for 4 GRB hosts, the data are not deep enough to judge whether they are molecule deficient (000418, 030329, 031203, and 090423). The median value of the molecular gas depletion time, $M_{\mathrm{H}_{2}} / \mathrm{SFR}$, of GRB hosts is $\sim 0.3$ dex below that of other star-forming galaxies, but this result has low statistical significance. A Kolmogorov-Smirnov test performed on $M_{\mathrm{H}_{2}} /$ SFR shows only $\sim 2 \sigma$ difference between GRB hosts and other galaxies. This difference can partially be explained by metallicity effects, since the significance decreases to $\sim 1 \sigma$ for $M_{\mathrm{H}_{2}} /$ SFR versus metallicity.

We found that any molecular gas deficiency of GRB hosts has low statistical significance and that it can be attributed to their lower metallicities; and thus the sample of GRB hosts has consistent molecular properties to other galaxies and can be treated as representative of star-forming galaxies. However, the molecular gas deficiency can be strong for GRB hosts if they exhibit higher excitations and/or a lower CO-to- $\mathrm{H}_{2}$ conversion factor than we assume, which would lead to lower molecular gas masses than we derive. Given the concentration of atomic gas 
recently found close to GRB and SN sites, indicating recent gas inflow, our results about the weak molecular deficiency imply that such inflow does not enhance the SFRs significantly, or that atomic gas converts efficiently into the molecular phase, which fuels star formation. Only if the analysis of a larger GRB host sample reveals molecular deficiency (especially close to the GRB position) would this support the hypothesis of star formation fuelled directly by atomic gas.

Acknowledgements. We thank Joanna Baradziej and our referee for help with improving this paper, Per Bergman, Carlos De Breuck, Palle Møller and Katharina Immer for help with the APEX observations, and Claudia Marka for help with IRAM 30 m observations. M.J.M. acknowledges the support of the National Science Centre, Poland through the POLONEZ grant 2015/19/P/ST9/04010 and the UK Science and Technology Facilities Council; this project has received funding from the European Union's Horizon 2020 research and innovation programme under the Marie Skłodowska-Curie grant agreement No. 665778. A.K. acknowledges support from the Polish National Science Center grants 2014/15/B/ST9/02111and 2016/21/D/ST9/01098. J.R.R. acknowledges the support from project ESP2015-65597-C4-1-R (MINECO/FEDER). A.J.C.T. acknowledges support from the Spanish Ministry Project AYA2015 71718-R. J.H. was supported by a VILLUM FONDEN Investigator gran (project number 16599). L.K.H. acknowledges funding from the INAF PRIN SKA program 1.05.01.88.04. M.R.K. acknowledges support from the Australian government through the Australian Research Council's Discovery Projects funding scheme (project DP160100695). Based on observations collected at the European Organisation for Astronomical Research in the Southern Hemisphere under ESO programmes 096.D-0280(A), 096.F-9302(A), and 097.F-9308(A) This publication is based on data acquired with the Atacama Pathfinder Experiment (APEX). APEX is a collaboration between the Max-Planck-Institut fur Radioastronomie, the European Southern Observatory, and the Onsala Space Observatory. This work is based on observations carried out under project number 172-16 with the IRAM $30 \mathrm{~m}$ telescope. IRAM is supported by INSU/CNRS (France), MPG (Germany) and IGN (Spain). This research has made use of the GHostS database (http: //www.grbhosts.org), which is partly funded by Spitzer/NASA grant RSA Agreement No. 1287913; the NASA/IPAC Extragalactic Database (NED) which is operated by the Jet Propulsion Laboratory, California Institute of Technology, under contract with the National Aeronautics and Space Administration; SAOImage DS9, developed by Smithsonian Astrophysical Observatory (Joye \& Mandel 2003); and the NASA's Astrophysics Data System Bibliographic Services.

\section{References}

Arabsalmani, M., Roychowdhury, S., Zwaan, M. A., Kanekar, N., \& Michałowski, M. J. 2015, MNRAS, 454, L51

Arabsalmani, M., Le Floc'h, E., Dannerbauer, H., et al. 2018, MNRAS, 476, 2332

Belitsky, V., Lapkin, I., \& Monje, R. 2006, in Proc. SPIE Conf. Ser., 6275, $62750 \mathrm{G}$

Belitsky, V., Lapkin, I., Fredrixon, M., et al. 2018, A\&A, 612, A23

Bertemes, C., Wuyts, S., Lutz, D., et al. 2018, MNRAS, 478, 1442

Bigiel, F., Leroy, A., Walter, F., et al. 2010, AJ, 140, 1194

Bloom, J. S., Berger, E., Kulkarni, S. R., Djorgovski, S. G., \& Frail, D. A. 2003 AJ, 125, 999

Boissier, S., Salvaterra, R., Le Floc'h, E., et al. 2013, A\&A, 557, A34

Bolatto, A. D., Wolfire, M., \& Leroy, A. K. 2013, ARA\&A, 51, 207

Boselli, A., Eales, S., Cortese, L., et al. 2010, PASP, 122, 261

Boselli, A., Cortese, L., \& Boquien, M. 2014, A\&A, 564, A65

Bothwell, M. S., Smail, I., Chapman, S. C., et al. 2013, MNRAS, 429, 3047

Bothwell, M. S., Wagg, J., Cicone, C., et al. 2014, MNRAS, 445, 2599

Butler, N. R., Bloom, J. S., \& Poznanski, D. 2010, ApJ, 711, 495

Carilli, C. L., \& Walter, F. 2013, ARA\&A, 51, 105

Carter, M., Lazareff, B., Maier, D., et al. 2012, A\&A, 538, A89

Castro Cerón, J. M., Michałowski, M. J., \& Hjorth, J., 2006, ApJ, 653, L85

Castro Cerón, J. M., Hjorth, M. J., \& Michałowski, J., 2010, ApJ, 721, 1919

Castro-Tirado, A. J., Bremer, M., McBreen, S., et al. 2007, A\&A, 475, 101

Chabrier, G. 2003, ApJ, 586, L133

Christensen, L., Vreeswijk, P. M., Sollerman, J., et al. 2008, A\&A, 490, 45

Ciesla, L., Boquien, M., Boselli, A., et al. 2014, A\&A, 565, A128

Cormier, D., Madden, S. C., Lebouteiller, V., et al. 2014, A\&A, 564, A121

Cortese, L., Boissier, S., Boselli, A., et al. 2012, A\&A, 544, A101

Cortese, L., Fritz, J., Bianchi, S., et al. 2014, MNRAS, 440, 942

Daddi, E., Bournaud, F., Walter, F., et al. 2010, ApJ, 713, 686
Davies, L. J. M., Driver, S. P., Robotham, A. S. G., et al. 2016, MNRAS, 461, 458

Devereux, N. A., \& Young, J. S. 1990, ApJ, 359, 42

Elliott, J., Greiner, J., Khochfar, S., et al. 2012, A\&A, 539, A113

Elmegreen, B. G. 2018, ApJ, 854, 16

Elmegreen, B. G., Kaufman, M., Bournaud, F., et al. 2016, ApJ, 823, 26

Endo, A., Kohno, K., Hatsukade, B., et al. 2007, ApJ, 659, 1431

Fumagalli, M., \& Gavazzi, G. 2008, A\&A, 490, 571

Fumagalli, M., Krumholz, M. R., Prochaska, J. X., Gavazzi, G., \& Boselli, A. 2009, ApJ, 697, 1811

Galbany, L., Mora, L., González-Gaitán, S., et al. 2017, MNRAS, 468, 628

Glover, S. C. O., \& Clark, P. C. 2012, MNRAS, 421, 9

Greiner, J., Peimbert, M., \& Esteban, C., 2003, GRB Coordinates Network, 2020

Greiner, J., Fox, D. B., Schady, P., et al. 2015, ApJ, 809, 76

Greiner, J., Michałowski, M. J., Klose, S., et al. 2016, A\&A, 593, A17

Grossi, M., Corbelli, E., Bizzocchi, L., et al. 2016, A\&A, 590, A27

Güsten, R., Nyman, L. A., Schilke, P., et al. 2006, A\&A, 454, L13

Hammer, F., Flores, H., Schaerer, D., et al. 2006, A\&A, 454, 103

Hatsukade, B., Kohno, K., Endo, A., et al. 2007, PASJ, 59, 67

Hatsukade, B., Kohno, K., Endo, A., Nakanishi, K., \& Ohta, K. 2011, ApJ, 738, 33

Hatsukade, B., Ohta, K., Endo, A., et al. 2014, Nature, 510, 247

Hjorth, J., \& Bloom, J. S. 2012, in Gamma-Ray Bursts, eds. C. Kouveliotou,

R. A. M. J. Wijers, \& S. Woosley (Cambridge: Cambridge Univ. Press), 169

Hjorth, J., Sollerman, J., Møller, P., et al. 2003, Nature, 423, 847

Hjorth, J., Malesani, D., Jakobsson, P., et al. 2012, ApJ, 756, 187

Hu, C. Y., Naab, T., Walch, S., Glover, S. C. O., \& Clark, P. C. 2016, MNRAS, 458,3528

Huang, S., Haynes, M. P., Giovanelli, R., et al. 2012, AJ, 143, 133

Hunt, L., Palazzi, E., Rossi, A., et al. 2011, ApJ, 736, L36

Hunt, L. K., Palazzi, E., Michałowski, M. J., et al. 2014a, A\&A, 565, A112

Hunt, L. K., Testi, L., Casasola, V., et al. 2014b, A\&A, 561, A49

Hunt, L. K., García-Burillo, S., Casasola, V., et al. 2015, A\&A, 583, A114

Hunt, L. K., Weiß, A., Henkel, C., et al. 2017, A\&A, 606, A99

Joye, W. A., \& Mandel, E. 2003, in Astronomical Data Analysis Software and Systems XII, eds. H. E. Payne, R. I. Jedrzejewski, \& R. N. Hook, ASP Conf. Ser., 295, 489

Kennicutt, R. C. 1998, ARA\&A, 36, 189

Kewley, L. J., \& Dopita, M. A. 2002, ApJS, 142, 35

Kewley, L. J., \& Ellison, S. L. 2008, ApJ, 681, 1183

Kistler, M. D., Yüksel, H., Beacom, J. F., Hopkins, A. M., \& Wyithe, J. S. B. 2009, ApJ, 705, L104

Kobulnicky, H. A., \& Kewley, L. J. 2004, ApJ, 617, 240

Kohn, S. A., Michałowski, M. J., Bourne, N., et al. 2015, MNRAS, 448, 1494

Kohno, K., Tosaki, T., Okuda, T., et al. 2005, PASJ, 57, 147

Krühler, T., Malesani, D., Fynbo, J. P. U., et al. 2015, A\&A, 581, A125

Krühler, T., Kuncarayakti, H., Schady, P., et al. 2017, A\&A, 602, A85

Krumholz, M. R. 2012, ApJ, 759, 9

Le Floc'h, E., Charmandaris, V., Forrest, W. J., et al. 2006, ApJ, 642, 636

Le Floc'h, E., Charmandaris, V., Gordon, K., et al. 2012, ApJ, 746, 7

Lee, J. C., Gil de Paz, A., \& Tremonti, C. 2009, ApJ, 706, 599

Leroy, A., Cannon, J., Walter, F., Bolatto, A., \& Weiss, A. 2007, ApJ, 663, 990

Leroy, A. K., Walter, F., Brinks, E., et al. 2008, AJ, 136, 2782

Levan, A. J., Tanvir, N. R., Wiersema, K., \& O'Brien, P. T., 2011, GRB Coordinates Network, 12414

Levesque, E. M., Berger, E., Kewley, L. J., \& Bagley, M. M. 2010, AJ, 139, 694

Levesque, E. M., Berger, E., Soderberg, A. M., \& Chornock, R. 2011, ApJ, 739, 23

Madden, S. C., Rémy-Ruyer, A., Galametz, M., et al. 2013, PASP, 125, 600

Magdis, G. E., Daddi, E., Elbaz, D., et al. 2011, ApJ, 740, L15

Magnelli, B., Saintonge, A., Lutz, D., et al. 2012, A\&A, 548, A22

Mazzali, P. A., Deng, J., Pian, E., et al. 2006, ApJ, 645, 1323

Michałowski, M. J., Hjorth, J., Castro Cerón, J. M., \& Watson, D. 2008, ApJ, 672,817

Michałowski, M. J., Hjorth, J., Malesani, D., et al. 2009, ApJ, 693, 347

Michałowski, M. J., Hjorth, J., \& Watson, D. 2010, A\&A, 514, A67

Michałowski, M. J., Kamble, A., Hjorth, J., et al. 2012, ApJ, 755, 85

Michałowski, M. J., Hunt, L. K., Palazzi, E., et al. 2014, A\&A, 562, A70

Michałowski, M. J., Gentile, G., Hjorth, J., et al. 2015, A\&A, 582, A78

Michałowski, M. J., Castro Cerón, J. M., \& Wardlow, J. L. 2016, A\&A, 595, A72

Michałowski, M. J., Xu, D., Stevens, J., et al., 2018a, A\&A, 616, A169

Michałowski, M. J., Gentile, G., \& Krühler, T., 2018b, A\&A, in press, DOI 10.1051/0004-6361/201732356

Ofek, E. O., Cenko, S. B. \& Gal-Yam, A. 2006, GRB Coordinates Network, 5123

Perley, D. A., Levan, A. J., Tanvir, N. R., et al. 2013, ApJ, 778, 128

Perley, D. A., Perley, R. A., Hjorth, J., et al. 2015, ApJ, 801, 102

Perley, D. A., Krühler, T., Schulze, S., et al. 2016a, ApJ, 817, 7

Perley, D. A., Tanvir, N. R., Hjorth, J., et al. 2016b, ApJ, 817, 8 
Perley, D. A., Hjorth, J., Tanvir, N. R., \& Perley, R. A. 2017a, MNRAS, 465, 970 Perley, D. A., Krühler, T., Schady, P., et al. 2017b, MNRAS, 465, L89 Pettini, M., \& Pagel, B. E. J. 2004, MNRAS, 348, L59

Pety, J. 2005, in SF2A-2005: Semaine de l'Astrophysique Francaise, eds. F. Casoli, T. Contini, J. M. Hameury, \& L. Pagani, 721

Pignata, G., Maza, J., \& Hamuy, M. 2009, Central Bureau Electronic Telegrams, 1731

Prochaska, J. X., Bloom, J. S., Chen, H. W., et al. 2004, ApJ, 611, 200

Rafelski, M., Gardner, J. P., Fumagalli, M., et al. 2016, ApJ, 825, 87

Robertson, B. E., \& Ellis, R. S. 2012, ApJ, 744, 95

Saintonge, A., Kauffmann, G., Kramer, C., et al. 2011, MNRAS, 415, 32

Salim, S., Rich, R. M., Charlot, S., et al. 2007, ApJs, 173, 267

Salpeter, E. E. 1955, ApJ, 121, 161

Salvaterra, R., Della-Valle M., Campana, S., et al. 2009, Nature, 461, 1258

Sanders, D. B., Scoville, N. Z., \& Soifer, B. T. 1991, ApJ, 370, 158

Savaglio, S., Glazebrook, K., \& LeBorgne, D. 2009, ApJ, 691, 182

Schady, P., Savaglio, S., Müller, T., et al. 2014, A\&A, 570, A52

Schulze, S., Chapman, R., Hjorth, J., et al. 2015, ApJ, 808, 73

Sollerman, J., Östlin, G., Fynbo, J. P. U., et al. 2005, New Astron., 11, 103

Solomon, P. M., Downes, D., Radford, S. J. E., \& Barrett, J. W. 1997, ApJ, 478, 144

Spring, E. F., \& Michałowski, M. J. 2017, MNRAS, 471, L101

Stanek, K. Z., Matheson, T., Garnavich, P. M., et al. 2003, ApJ, 591, L17

Stanway, E. R., Bremer, M. N., Tanvir, N. R., Levan, A. J., \& Davies, L. J. M. 2011, MNRAS, 410, 1496

Stanway, E. R., Levan, A. J., Tanvir, N., et al. 2015a, MNRAS, 446, 3911
Stanway, E. R., Levan, A. J., Tanvir, N. R., Wiersema, K., \& van der Laan, T. P. R. 2015b, ApJ, 798, L7

Starling, R. L. C., Wiersema, K., Levan, A. J., et al. 2011, MNRAS, 411, 2792

Svensson, K. M., Levan, A. J., Tanvir, N. R., Fruchter, A. S., \& Strolger, L. 2010, MNRAS, 405, 57

Svensson, K. M., Levan, A. J., Tanvir, N. R., et al. 2012, MNRAS, 421, 25

Tanga, M., Krühler, T., \& Schady, P. 2018, A\&A, 615, A136

Tanvir, N. R., Fox, D. B., Levan, A. J., et al. 2009, Nature, 461, 1254

Thöne, C. C., Fynbo, J. P. U., Östlin, G., et al. 2008, ApJ, 676, 1151

Thöne, C. C., Michałowski, M. J., Leloudas, G., et al. 2009, ApJ, 698, 1307

Tinney, C., Stathakis, R., Cannon, R., 1998. IAU Circ., 6896

Vassilev, V., Meledin, D., Lapkin, I., et al. 2008, A\&A, 490, 1157

Vergani, S. D., D’Avanzo, P., Levan, A. J., 2010, GRB Coordinates Network, 10512

Vergani, S. D., Salvaterra, R., Japelj, J., et al. 2015, A\&A, 581, A102

Walter, F., Decarli, R., Carilli, C., et al. 2012, ApJ, 752, 93

Wang, L., Norberg, P., Gunawardhana, M. L. P., et al. 2016, MNRAS, 461, 1898

Watson, D., French, J., Christensen, L., et al. 2011, ApJ, 741, 58

Wiersema, K., Togi, A., Watson, D., et al., 2018, MNRAS, 481, 1126

Wijesinghe, D. B., da Cunha, E., Hopkins, A. M., et al. 2011, MNRAS, 415, 1002

Young, J. S., Xie, S., Kenney, J. D. P., \& Rice, W. L. 1989, ApJs, 70, 699

Yüksel, H., Kistler, M. D., Beacom, J. F., \& Hopkins, A. M. 2008, ApJ, 683, L5

\section{Appendix A: Additional table}

Table A.1. Properties of our sample of GRB hosts.

\begin{tabular}{|c|c|c|c|c|c|c|}
\hline GRB & $z_{\text {opt }}$ & Ref. & $\begin{array}{c}\mathrm{SFR} \\
\left(M_{\odot} \mathrm{yr}^{-1}\right)\end{array}$ & Ref. & $12+\log (\mathrm{O} / \mathrm{H})$ & Ref. \\
\hline 980425 & 0.0085 & Tinney et al. (1998) & 0.26 & Michałowski et al. (2014) & 8.60 & Sollerman et al. (2005) \\
\hline 980425_WR & 0.0085 & Tinney et al. (1998) & 0.02 & Michałowski et al. (2014) & 8.16 & Christensen et al. (2008) \\
\hline 000418 & 1.1181 & Bloom et al. (2003) & $<77$ & Perley et al. (2017a) & 8.43 & Svensson et al. (2010) \\
\hline 030329 & 0.1685 & Greiner et al. (2003), Hjorth et al. (2003) & $<17$ & Michałowski et al. (2012) & 8.13 & Levesque et al. (2010) \\
\hline 031203 & 0.1050 & Prochaska et al. (2004) & 2.8 & Watson et al. (2011) & 8.27 & Levesque et al. (2010) \\
\hline 051022 & 0.809 & Castro-Tirado et al. (2007) & 17.9 & Hunt et al. (2014a) & 8.62 & Levesque et al. (2010) \\
\hline 060505 & 0.0889 & Ofek et al. (2006) & 0.69 & Michałowski et al. (2015) & 8.30 & Thöne et al. (2008) \\
\hline 060814 & 1.9229 & Hjorth et al. (2012) & 256 & Perley et al. (2015) & 8.38 & Krühler et al. (2015) \\
\hline 080207 & 2.0858 & Hjorth et al. (2012) & 170 & Hunt et al. (2014a) & 8.74 & Krühler et al. (2015) \\
\hline 080517 & 0.089 & Stanway et al. (2015a) & 7.6 & Stanway et al. (2015a) & 8.66 & Stanway et al. (2015a) \\
\hline 090423 & 8.23 & Tanvir et al. (2009), Salvaterra et al. (2009) & $<39$ & Walter et al. (2012) & - & - \\
\hline $100316 \mathrm{D}$ & 0.0591 & Vergani et al. (2010), Starling et al. (2011) & 1.73 & Michałowski et al. (2015) & 8.30 & Levesque et al. (2011) \\
\hline $111005 \mathrm{~A}$ & 0.01326 & Michałowski et al. (2018a), Levan et al. (2011) & 0.42 & Michałowski et al. (2018a) & 8.50 & Tanga et al. (2018) \\
\hline 111005A_CENT & 0.01326 & Michałowski et al. (2018a), Levan et al. (2011) & 0.26 & Tanga et al. (2018) & 8.56 & Tanga et al. (2018) \\
\hline 111005A_NW & 0.01326 & Michałowski et al. (2018a), Levan et al. (2011) & 0.06 & Tanga et al. (2018) & 8.49 & Tanga et al. (2018) \\
\hline 111005A_SE & 0.01326 & Michałowski et al. (2018a), Levan et al. (2011) & 0.09 & Tanga et al. (2018) & 8.43 & Tanga et al. (2018) \\
\hline SN 2009bb & 0.009877 & Pignata et al. (2009) & 5.21 & Michałowski et al. (2018b) & 8.66 & Michałowski et al. (2018b) \\
\hline
\end{tabular}

